\title{
From axioms to analytic rules in nonclassical logics
}

\author{
Agata Ciabattoni* \\ Vienna University of Technology \\ agata@logic.at \\ Kazushige Terui \\ Nikolaos Galatos \\ University of Denver \\ ngalatos@du.edu \\ National Institute of Informatics, Japan \\ Laboratoire d'Informatique de Paris Nord, CNRS \\ terui@nii.ac.jp
}

\begin{abstract}
We introduce a systematic procedure to transform large classes of (Hilbert) axioms into equivalent inference rules in sequent and hypersequent calculi. This allows for the automated generation of analytic calculi for a wide range of propositional nonclassical logics including intermediate, fuzzy and substructural logics. Our work encompasses many existing results, allows for the definition of new calculi and contains a uniform semantic proof of cutelimination for hypersequent calculi.
\end{abstract}

\section{Introduction}

Nonclassical logics are often presented by extending with suitable axioms the (Hilbert) calculi of well known systems. The applicability/usefulness of these logics, however, strongly depends on the availability of analytic calculi. Such calculi, where proof search proceeds by step-wise decomposition of the formula to be proved, are not only useful in establishing important properties of corresponding logics, such as decidability or the Herbrand theorem, but also the key to develop automated reasoning methods.

Since its introduction by Gentzen in [7], sequent calculus has been one of the preferred frameworks to define analytic calculi. This framework is however not capable of handling all interesting and useful logics. A large range of variants and extensions of sequent calculus have been indeed introduced in the last few decades to define analytic calculi for logics that seemed to escape a (cut-free) sequent formalisation; a prominent example being Gödel logic, obtained by extending intuitionistic logic IL with the prelinearity axiom $(\alpha \rightarrow \beta) \vee(\beta \rightarrow \alpha)$. An analytic calculus for this logic was defined by Avron using hypersequents [2] - a simple

\footnotetext{
* Research supported by FWF Project P18731.
}

generalization of Gentzen sequents to multisets of sequents. Since then hypersequent calculi have been discovered for a wide range of nonclassical logics, e.g. [2, 3, 4, 5, 11]. This is traditionally done by (i) looking for the "right" inference rule(s) formalizing the particular properties of the logic under consideration (e.g., Avron introduced the rule (com), corresponding to the prelinearity axiom) and (ii) proving cut-elimination (or cut-admissibility) to show that the resulting calculus is analytic. These two steps are usually tailored to the particular logic at hand, and each calculus needs its own proof of soundness, completeness and cutelimination. This holds even when adding the same rules to different base calculi (e.g. IL + (com) dealt with in [2], IL $+(\mathrm{com})$ - contraction in [4], and IL $+(\mathrm{com})$ - weakening - contraction in [11]), which might cause a combinatorial explosion on the number of the papers to be produced.

In this paper we introduce a systematic procedure for performing step (i) and a uniform (semantic) proof for step (ii) for a wide range of logics extending $\mathbf{F L e}^{1}$, i.e., intuitionistic linear logic without exponentials. This allows for the automated generation of analytic calculi for a wide range of nonclassical logics including intermediate, substructural and fuzzy logics.

More precisely, we define a hierarchy - analogous to the arithmetical hierarchy $\Sigma_{n}, \Pi_{n}$ - over the formulas of FLe and show how to translate the axioms at levels up to $\mathcal{N}_{2}$ (resp. up to $\mathcal{P}_{3}$ ) into equivalent structural sequent rules (resp. hypersequent rules). See Figure 2 for examples of axioms, considered in the literature of intermediate, substructural and fuzzy logics, that fall into these two classes. When the generated rules satisfy an additional condition or the base calculus admits weakening, these are further transformed (completed) into equivalent analytic rules, i.e., they preserve cut-elimination once added to FLe. The analyticity of the generated calculi is proved once and for all by

\footnotetext{
${ }^{1}$ FLe stands for Full Lambek calculus with exchange, see e.g. [8].
} 
extending Okada's semantic proof of cut-elimination [13] to hypersequent calculi. The completion procedure sheds also light on the expressive power and limitations of structural (hyper)sequent rules.

Our work accounts uniformly for many existing results, and new ones can be generated in an automated way. For instance, by applying our procedure a first analytic calculus is found for Weak Nilpotent Minimum Logic WNM [6] the logic of left-continuous $t$-norms ${ }^{2}$ satisfying the weak nilpotent minimum property (Corollary 8.9).

\section{Preliminaries}

The base calculus we will deal with is the sequent system FLe i.e., Full Lambek calculus FL extended with exchange (see e.g. [8]). Roughly speaking, FLe is obtained by dropping the structural rules of weakening (w) and contraction (c) from the sequent calculus LJ (FLewc, in our terminology) for intuitionistic logic. Also, FLe is the same as intuitionistic linear logic without exponentials.

The formulas of FLe are built from propositional variables $p, q, r, \ldots$ and the 0 -ary connectives (constants) 1 (unit), $\perp$ (false), $\top$ (true) and 0 by using the binary logical connectives $\cdot$ (fusion), $\rightarrow$ (implication), $\wedge$ (conjunction) and $\vee$ (disjunction). $\neg \alpha$ and $\alpha \leftrightarrow \beta$ will be used as abbreviations for $\alpha \rightarrow 0$ and $(\alpha \rightarrow \beta) \wedge(\beta \rightarrow \alpha)$. (Our notation should not be confused with that of linear logic, where symbol 0 is used for $\perp$ and vice versa.)

Henceforth metavariables $\alpha, \beta, \ldots$ will denote formulas, $\Pi, \Theta$ will stand for stoups, i.e., either a formula or the empty set, and $\Gamma, \Delta, \ldots$ for finite (possibly empty) multisets of formulas. In this paper we will only consider sequents in the language of FLe that are single-conclusion, i.e., whose right-hand side (RHS) contains at most one formula. As usual, axioms and inference rules are specified by using metavariables together with metaformulas, i.e., expressions built from metavariables $\alpha, \beta, \ldots$ by using the logical connectives of FLe. See Fig. 1 for the inference rules of FLe.

An axiom (scheme) is a metaformula $\psi$, which we identify with a rule $\overline{\Rightarrow \psi}$ with 0 premises.

By structural rule we mean any sequent rule, with the exception of (init) and (cut), of the form $(n \geq 0)$

$$
\frac{\Upsilon_{1} \Rightarrow \Psi_{1} \quad \ldots \quad \Upsilon_{n} \Rightarrow \Psi_{n}}{\Upsilon_{0} \Rightarrow \Psi_{0}}(r)
$$

where each $\Upsilon_{i}$ is a specific multiset of metavariables allowed to be of both types: metavariables for formulas $(\alpha)$ or for multisets of formulas $(\Gamma)$, and each $\Psi_{i}$ is either empty, a metavariable for formulas, or a metavariable for stoups (П). Examples of structural rules are found in Figure 3.

\footnotetext{
${ }^{2} T$-norms are the main tool in fuzzy logic to combine vague information.
}

Notice that a metavariable is used in two ways: as a notation that stands for (possibly compound) concrete formulas and as an (atomic) building block for defining axioms and rules. We do not make a rigorous distinction between them, relying on the standard practice in our field.

The notion of proof in FLe is defined as usual. Let $R$ be a set of rules. If there is a proof in FLe extended with $R$ (FLe $+R$, for short) of a sequent $S_{0}$ from a set of sequents $\mathcal{S}$, we say that $S_{0}$ is derivable from $\mathcal{S}$ in $\mathbf{F L e}+R$ and write $\mathcal{S} \vdash_{\mathbf{F L e}+R} S_{0}$. We write $\vdash_{\mathbf{F L e}+R} \alpha$ if $\emptyset \vdash_{\mathbf{F L e}+R} \Rightarrow \alpha$.

The logical connectives of FLe are classified into two groups according to their polarities [1]: $1, \perp, \cdot, \vee$ (resp. $0, \top, \rightarrow, \wedge$ ) are positive (resp. negative) connectives for which the left (resp. right) logical rule is invertible, i.e., the conclusion implies the premises. E.g. we have for $(\vee l)$ :

- $\vdash_{\mathbf{F L e}} \alpha \vee \beta, \Gamma \Rightarrow \Pi$ iff $\vdash_{\mathbf{F L e}} \alpha, \Gamma \Rightarrow \Pi$ and $\vdash_{\mathbf{F L e}}$ $\beta, \Gamma \Rightarrow \Pi$.

Connectives of the same polarity interact well with each other:

$$
\begin{aligned}
& \text { (P) } \vdash_{\mathbf{F L e}} \alpha \cdot 1 \leftrightarrow \alpha, \alpha \vee \perp \leftrightarrow \alpha,(\alpha \cdot \perp) \leftrightarrow \perp, \\
& \alpha \cdot(\beta \vee \gamma) \leftrightarrow(\alpha \cdot \beta) \vee(\alpha \cdot \gamma) . \\
& (\mathrm{N}) \vdash_{\mathbf{F L e}} \alpha \wedge \top \leftrightarrow \alpha,(1 \rightarrow \alpha) \leftrightarrow \alpha,(\alpha \rightarrow \top) \leftrightarrow \top, \\
& (\alpha \rightarrow(\beta \wedge \gamma)) \leftrightarrow(\alpha \rightarrow \beta) \wedge(\alpha \rightarrow \gamma),(\perp \rightarrow \alpha) \leftrightarrow \top, \\
& ((\alpha \vee \beta) \rightarrow \gamma) \leftrightarrow(\alpha \rightarrow \gamma) \wedge(\beta \rightarrow \gamma) .
\end{aligned}
$$

(Notice that polarity is reversed on the left hand side of an implication. For instance, the $\vee$ on the left-hand side (LHS) of $\rightarrow$ in the last equivalence is considered negative.)

Since connectives $\wedge, \vee, \cdot$ have units $\top, \perp, 1$ respectively, we adopt a natural convention: $\beta_{1} \vee \cdots \vee \beta_{m}$ (resp. $\beta_{1} \wedge \cdots \wedge$ $\beta_{m}$ and $\beta_{1} \cdots \beta_{m}$ ) stands for $\perp$ (resp. $\top$ and 1$)$ if $m=0$.

We say that two formulas $\alpha$ and $\beta$ are (externally) equivalent in FLe if $\alpha \vdash_{\text {FLe }} \beta$ and $\beta \vdash_{\text {FLe }} \alpha$. Obviously $\vdash_{\text {FLe }} \alpha \leftrightarrow \beta$ implies that $\alpha$ and $\beta$ are equivalent, while the converse does not hold due to lack of a deduction theorem. A counterexample is that $\alpha$ and $\alpha \wedge 1$ are equivalent whereas $\nvdash_{\text {FLe }} \alpha \leftrightarrow \alpha \wedge 1$. In contrast with internal equivalence (i.e. $\vdash_{\text {FLe }} \alpha \leftrightarrow \beta$ ), external equivalence is not a congruence; indeed, $\alpha \vee \beta$ and $(\alpha \wedge 1) \vee \beta$ are not equivalent. If we are allowed to use the modality $! \alpha$ of linear logic, external equivalence can be internalized: $\vdash_{\mathbf{F L e}} ! \alpha \leftrightarrow ! \beta$.

Two rules $\left(r_{0}\right)$ and $\left(r_{1}\right)$ are equivalent (in FLe) if the relations $\vdash_{\mathbf{F L e}+\left(r_{0}\right)}$ and $\vdash_{\mathbf{F L e}+\left(r_{1}\right)}$ coincide. In particular, when the conclusion of $\left(r_{0}\right)$ (and resp. of $\left.\left(r_{1}\right)\right)$ is derivable from its premises in FLe $+\left(r_{1}\right)$ (resp. FLe $\left.+\left(r_{0}\right)\right)$ then $\left(r_{0}\right)$ and $\left(r_{1}\right)$ are equivalent. The definition naturally extends to sets of rules.

\section{Substructural Hierarchy}

To address systematically the problem of translating axioms into equivalent structural rules in an automated way 


\begin{tabular}{|c|c|c|c|}
\hline$\frac{G|\Gamma \Rightarrow \alpha \quad G| \alpha, \Delta \Rightarrow \Pi}{G \mid \Gamma, \Delta \Rightarrow \Pi}($ cut $)$ & $\overline{G \mid \alpha \Rightarrow \alpha}$ (init) & $\frac{G \mid \Gamma \Rightarrow \Pi}{G \mid 1, \Gamma \Rightarrow \Pi}(1 l)$ & $\overline{G \mid \Rightarrow 1}(1 r)$ \\
\hline$\frac{G \mid \alpha, \beta, \Gamma \Rightarrow \Pi}{G \mid \alpha \cdot \beta, \Gamma \Rightarrow \Pi}(\cdot l)$ & $\frac{G|\Gamma \Rightarrow \alpha \quad G| \Delta \Rightarrow \beta}{G \mid \Gamma, \Delta \Rightarrow \alpha \cdot \beta}(\cdot r)$ & $\frac{G \mid \Gamma \Rightarrow}{G \mid \Gamma \Rightarrow 0}(0 r)$ & $\overline{G \mid 0 \Rightarrow}(0 l)$ \\
\hline$\frac{G|\Gamma \Rightarrow \alpha \quad G| \beta, \Delta \Rightarrow \Pi}{G \mid \Gamma, \alpha \rightarrow \beta, \Delta \Rightarrow \Pi}(\rightarrow l)$ & $\frac{G \mid \alpha, \Gamma \Rightarrow \beta}{G \mid \Gamma \Rightarrow \alpha \rightarrow \beta}(\rightarrow r)$ & $\frac{G \mid \alpha_{i}, \Gamma \Rightarrow \Pi}{G \mid \alpha_{1} \wedge \alpha_{2}, \Gamma \Rightarrow \Pi}(\wedge l)$ & $\overline{G \mid \perp, \Gamma \Rightarrow \Pi}(\perp l)$ \\
\hline$\frac{G|\alpha, \Gamma \Rightarrow \Pi \quad G| \beta, \Gamma \Rightarrow \Pi}{G \mid \alpha \vee \beta, \Gamma \Rightarrow \Pi}(\vee l)$ & $\frac{G|\Gamma \Rightarrow \alpha \quad G| \Gamma \Rightarrow \beta}{G \mid \Gamma \Rightarrow \alpha \wedge \beta}(\wedge r)$ & $\frac{G \mid \Gamma \Rightarrow \alpha_{i}}{G \mid \Gamma \Rightarrow \alpha_{1} \vee \alpha_{2}}(\vee r)$ & $\overline{G \mid \Gamma \Rightarrow \top}(\top r)$ \\
\hline$\frac{G}{G \mid \Gamma \Rightarrow \Pi}$ & $(E W)$ & $\frac{G|\Gamma \Rightarrow \Pi| \Gamma=}{G \mid \Gamma \Rightarrow \Pi}$ & $\underline{\Pi}(E C)$ \\
\hline
\end{tabular}

Figure 1. Inference Rules of HFLe

we introduce below a hierarchy on the FLe formulas based on their polarities, which is analogous to the arithmetical hierarchy $\Sigma_{n}, \Pi_{n}$.

Definition 3.1. For each $n \geq 0$, the sets $\mathcal{P}_{n}, \mathcal{N}_{n}$ of (positive and negative) formulas are defined as follows:

(0) $\mathcal{P}_{0}=\mathcal{N}_{0}=$ the set of propositional variables.

(P1) $1, \perp$ and any formula $\alpha \in \mathcal{N}_{n}$ belong to $\mathcal{P}_{n+1}$.

(P2) If $\alpha, \beta \in \mathcal{P}_{n+1}$, then $\alpha \vee \beta, \alpha \cdot \beta \in \mathcal{P}_{n+1}$.

(N1) $0, \top$ and any formula $\alpha \in \mathcal{P}_{n}$ belong to $\mathcal{N}_{n+1}$.

(N2) If $\alpha, \beta \in \mathcal{N}_{n+1}$, then $\alpha \wedge \beta \in \mathcal{N}_{n+1}$.

(N3) If $\alpha \in \mathcal{P}_{n+1}$ and $\beta \in \mathcal{N}_{n+1}$, then $\alpha \rightarrow \beta \in \mathcal{N}_{n+1}$.

An axiom $\psi$ belongs to $\mathcal{P}_{n}$ (resp. $\mathcal{N}_{n}$ ) if this holds when its metavariables are instantiated with propositional variables.

Example 3.2. $\neg(\alpha \cdot \beta) \vee(\alpha \wedge \beta \rightarrow \alpha \cdot \beta)$ (weak nilpotent minimum [6] $) \in \mathcal{P}_{3}$ while Łukasiewicz axiom $((\alpha \rightarrow \beta) \rightarrow$ $\beta) \rightarrow((\beta \rightarrow \alpha) \rightarrow \alpha) \in \mathcal{N}_{3}$.

See Figure 2 for further examples.

\section{Theorem 3.3.}

1. Every FLe formula belongs to some $\mathcal{P}_{n}$ and $\mathcal{N}_{n}$.

2. $\mathcal{P}_{n} \subseteq \mathcal{P}_{n+1}$ and $\mathcal{N}_{n} \subseteq \mathcal{N}_{n+1}$ for every $n$.

Hence the classes $\mathcal{P}_{n}, \mathcal{N}_{n}$ constitute a hierarchy, which we call substructural hierarchy, of the following form:

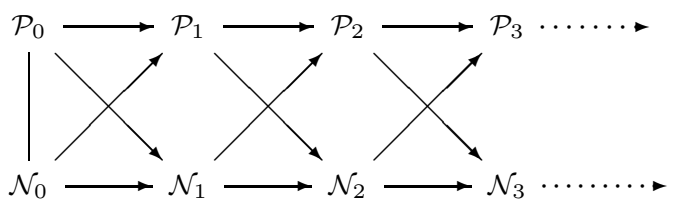

It is easy to see that formulas in each class admit the following normal forms:

\section{Lemma 3.4.}

(P) If $\alpha \in \mathcal{P}_{n+1}$, then we have $\vdash_{\mathbf{F L e}} \alpha \leftrightarrow \beta_{1} \vee \cdots \vee \beta_{m}$, where each $\beta_{i}$ is a fusion of formulas in $\mathcal{N}_{n}$.

(N) If $\alpha \in \mathcal{N}_{n+1}$, then $\vdash_{\text {FLe }} \alpha \leftrightarrow\left(\bigwedge_{1 \leq i \leq m} \gamma_{i} \rightarrow \beta_{i}\right)$, where each $\beta_{i}$ is either 0 or a formula in $\mathcal{P}_{n}$, and each $\gamma_{i}$ is a fusion of formulas in $\mathcal{N}_{n}$.

A formula $\alpha$ is said to be $\mathcal{N}_{2}$-normal if it is of the form $\alpha_{1} \cdots \alpha_{n} \rightarrow \beta$ where

- $\beta=0$ or $\beta_{1} \vee \cdots \vee \beta_{k}$ with each $\beta_{i}$ a fusion of propositional variables and

- each $\alpha_{i}$ is of the form $\bigwedge_{1 \leq j \leq m_{i}} \gamma_{i}^{j} \rightarrow \beta_{i}^{j}$, where $\beta_{i}^{j}=$ 0 or is a propositional variable, and $\gamma_{i}^{j}$ is a fusion of propositional variables.

As a consequence of the above lemma, every formula $\alpha$ in $\mathcal{N}_{2}$ can be transformed into a finite conjunction $\bigwedge_{1 \leq i \leq n} \alpha_{i}$ of $\mathcal{N}_{2}$-normal formulas such that $\vdash_{\text {FLe }} \alpha \leftrightarrow \bigwedge_{1 \leq i \leq n} \alpha_{i}$.

The lack of weakening in FLe makes it hard to deal with formulas in $\mathcal{P}_{3}$. We therefore introduce a subclass $\mathcal{P}_{3}^{\prime}$ of $\mathcal{P}_{3}$ defined as:

1. $1, \perp \in \mathcal{P}_{3}^{\prime}$

2. If $\alpha \in \mathcal{N}_{2}$ then $\alpha \wedge 1 \in \mathcal{P}_{3}^{\prime}$

3. If $\alpha, \beta \in \mathcal{P}_{3}^{\prime}$ then $\alpha \cdot \beta, \alpha \vee \beta \in \mathcal{P}_{3}^{\prime}$.

Henceforth we write $(\alpha)_{\wedge 1}$ for $\alpha \wedge 1$. 


\begin{tabular}{|c|c|c|c|c|}
\hline Class & Axiom & Name & Rule (cf. Fig. 3) & Reference \\
\hline$\overline{\mathcal{N}_{2}}$ & $\begin{array}{l}\alpha \rightarrow 1,0 \rightarrow \alpha \\
\alpha \rightarrow \alpha \cdot \alpha \\
\alpha \cdot \alpha \rightarrow \alpha \\
\alpha^{n} \rightarrow \alpha^{m} \\
\neg(\alpha \wedge \neg \alpha)\end{array}$ & $\begin{array}{c}\text { weakening } \\
\text { contraction } \\
\text { expansion } \\
\text { knotted axioms }(n, m \geq 0) \\
\text { weak contraction }\end{array}$ & $\begin{array}{c}(w),\left(w^{\prime}\right) \\
(c) \\
(\text { mingle }) \\
\left(\text { knot }_{m}^{n}\right) \\
(w c)\end{array}$ & $\begin{array}{c}{[7]} \\
{[7]} \\
{[12]} \\
{[9,16]^{* 2}}\end{array}$ \\
\hline$\overline{\mathcal{P}_{2}}$ & $\begin{array}{c}\alpha \vee \neg \alpha \\
(\alpha \rightarrow \beta) \vee(\beta \rightarrow \alpha)\end{array}$ & $\begin{array}{l}\text { excluded middle } \\
\text { prelinearity }\end{array}$ & $\begin{array}{c}(\mathrm{em}) \\
(\mathrm{com})^{* 1}\end{array}$ & $\begin{array}{l}3] \\
{[2]}\end{array}$ \\
\hline $\mathcal{P}_{3}^{\prime}$ & $((\alpha \rightarrow \beta) \wedge 1) \vee((\beta \rightarrow \alpha) \wedge 1)$ & linearity & $(\mathrm{com})$ & {$[2,4,11]^{* 3}$} \\
\hline $\mathcal{P}_{3}$ & $\begin{array}{c}\neg \alpha \vee \neg \neg \alpha \\
\bigvee_{i=0}^{k}\left(p_{i} \rightarrow \bigvee_{j \neq i} p_{j}\right) \\
p_{0} \vee\left(p_{0} \rightarrow p_{1}\right) \vee \cdots \vee\left(p_{0} \wedge \cdots \wedge p_{k-1} \rightarrow p_{k}\right)\end{array}$ & $\begin{array}{l}\text { weak excluded middle } \\
\text { Kripke models with width } \leq k \\
\text { Kripke models with } k \text { worlds }\end{array}$ & $\begin{array}{l}(l q)^{*_{1}} \\
(B w k)^{*_{1}} \\
(B c k)^{*_{1}}\end{array}$ & $\begin{array}{l}{[5]} \\
{[5]} \\
{[5]}\end{array}$ \\
\hline
\end{tabular}

$*_{1}$ : The rule is equivalent to the axiom in FLew.

$*_{2}$ : The rules $\left(k n o t_{m}^{n}\right)$ arise by applying the completion procedure in [18] to the rules in [16, 9].

$*_{3}$ : The rule (com) is due to [2] (added to IL). Later added to FLew by [4] and to FLe by [11].

\section{Figure 2. Axioms vs (hyper)sequent rules}

Lemma 3.5. Every formula in $\mathcal{P}_{3}^{\prime}$ is equivalent to a finite set of formulas $\left(\alpha_{1}\right)_{\wedge 1} \vee \cdots \vee\left(\alpha_{n}\right)_{\wedge 1}$ where each $\alpha_{i}$ is $\mathcal{N}_{2}$ normal.

Proof. We may assume that any formula $\psi \in \mathcal{P}_{3}^{\prime}$ is built from conjunctions of $\mathcal{N}_{2}$-normal formulas by clauses 2 and 3. $\psi$ can be transformed into the desired sets by applying the equivalences between

- $\left(\alpha_{\wedge 1} \cdot \beta_{\wedge 1}\right) \vee \gamma$ and the set $\left\{\alpha_{\wedge 1} \vee \gamma, \beta_{\wedge 1} \vee \gamma\right\}$,

- $(\alpha \wedge \beta)_{\wedge 1} \vee \gamma$ and $\left\{\alpha_{\wedge 1} \vee \gamma, \beta_{\wedge 1} \vee \gamma\right\}$

where $\gamma$ is any formula in $\mathcal{P}_{3}^{\prime}$. To prove these equivalences we use the fact that $\alpha_{\wedge 1} \vee \gamma, \beta_{\wedge 1} \vee \gamma \Rightarrow\left(\alpha_{\wedge 1} \cdot \beta_{\wedge 1}\right) \vee \gamma$ and $\left(\alpha_{\wedge 1} \cdot \beta_{\wedge 1}\right) \vee \gamma \Rightarrow(\alpha \wedge \beta)_{\wedge 1} \vee \gamma$ are provable in FLe.

Notice that in presence of weakening (i.e., in FLew) Lemma 3.5 holds for every formula in $\mathcal{P}_{3}$, as $\vdash_{\text {FLew }} \alpha \leftrightarrow$ $\alpha_{\wedge 1}$.

\section{4. $\mathcal{N}_{2}$ and sequent rules}

We provide a systematic procedure for transforming any axiom in $\mathcal{N}_{2}$ into a set of equivalent structural rules. The key observation being

Lemma 4.1. The rule $\frac{S_{1} \cdots S_{m}}{\psi_{1}, \ldots, \psi_{n} \Rightarrow \xi}(r)$ is equivalent to each of the rules

$$
\frac{\vec{S} \quad \alpha_{1} \Rightarrow \psi_{1} \quad \cdots \quad \alpha_{n} \Rightarrow \psi_{n}}{\alpha_{1}, \ldots, \alpha_{n} \Rightarrow \xi}\left(r_{1}\right) \frac{\vec{S} \quad \xi \Rightarrow \beta}{\psi_{1}, \ldots, \psi_{n} \Rightarrow \beta}\left(r_{2}\right)
$$

where $\vec{S}=S_{1} \cdots S_{m}$ and $\alpha_{1}, \ldots, \alpha_{n}, \beta$ are fresh metavariables for formulas.
Proof. The left-to-right direction follows by $(c u t)$. For instance, assume the premises of $\left(r_{2}\right)$. Then $\psi_{1}, \ldots, \psi_{n} \Rightarrow \xi$ follows from $\vec{S}$ by $(r)$. One can then apply (cut) to obtain the conclusion of $\left(r_{2}\right)$. For the right-to-left direction we instantiate $\alpha_{i}$ with $\psi_{i}(i=1, \ldots, n)$ and $\beta$ with $\xi$.

Theorem 4.2. Every axiom in $\mathcal{N}_{2}$ is equivalent to a finite set of structural rules.

Proof. By Lemma 3.4, it suffices to consider $\mathcal{N}_{2}$-normal axioms $\psi$. Let $\psi$ be $\psi_{1} \cdots \psi_{n} \rightarrow \xi$ with $\psi_{i}=\bigwedge_{1 \leq j \leq m_{i}} \phi_{i}^{j} \rightarrow$ $\xi_{i}^{j}$ for $i=1, \ldots, n$. By the invertibility of $(\rightarrow r),(\cdot l),(1 l)$ and Lemma 4.1 follows that $\psi_{1} \cdots \psi_{n} \rightarrow \xi$ is equivalent to the rule

$$
\frac{\alpha_{1} \Rightarrow \psi_{1} \quad \cdots \quad \alpha_{n} \Rightarrow \psi_{n}}{\alpha_{1}, \ldots, \alpha_{n} \Rightarrow \xi}
$$

with $\alpha_{1}, \ldots, \alpha_{n}$ fresh metavariables. The invertibility of $(\wedge r),(\rightarrow r),(\cdot l),(1 l)$ and $(0 r)$ allows us to replace the premises with an equivalent set $\mathcal{S}$ of sequents that consist of metavariables only. If $\xi=0$, then remove it from the conclusion to obtain a structural rule. Otherwise, $\xi=\xi_{1} \vee$ $\cdots \vee \xi_{k}$. By Lemma 4.1 and the invertibility of $(\vee l)$ follows that the above rule is equivalent to

$$
\frac{\mathcal{S} \quad \xi_{1} \Rightarrow \beta, \cdots \quad \xi_{k} \Rightarrow \beta}{\alpha_{1}, \ldots, \alpha_{n} \Rightarrow \beta}
$$

with $\beta$ fresh metavariable. The claim follows by the invertibility of $(\cdot l)$ and $(1 l)$.

Example 4.3. Using the algorithm described in the proof of the theorem above, axiom $\neg(\alpha \wedge \neg \alpha)$ (weak contraction, see Figure 2) is transformed into an equivalent structural rule as 
follows:

$$
\begin{aligned}
\overline{\Rightarrow \neg(\alpha \wedge \neg \alpha)} & \longrightarrow \frac{\overline{(\alpha \wedge \neg \alpha) \Rightarrow}}{\beta} \\
& \frac{\beta \Rightarrow \alpha \wedge \neg \alpha}{\beta \Rightarrow} \\
& \longrightarrow \frac{\beta \Rightarrow \alpha \quad \alpha, \beta \Rightarrow}{\beta \Rightarrow}
\end{aligned}
$$

A further transformation (called completion), described in Section 6, makes it into the analytic rule $(w c)$ in Fig. 3.

\section{5. $\mathcal{P}_{3}$ and hypersequent rules}

Consider some axiom beyond $\mathcal{N}_{2}$ such as weak excluded middle $\neg \alpha \vee \neg \neg \alpha$ and prelinearity $(\alpha \rightarrow \beta) \vee(\beta \rightarrow \alpha)$, see Fig. 2. Since they are neither valid nor contradictory in intuitionistic logic, Corollary 7.2 ensures that no structural rule added to FLe is equivalent to them. These axioms have been instead formalized in a natural way by structural rules in hypersequent calculus - a simple generalization of sequent calculus due to Avron (see [3] for an overview). We show below that this holds for all axioms in the class $\mathcal{P}_{3}^{\prime}$ ( $\mathcal{P}_{3}$, in presence of weakening) and we provide an algorithm for transforming any such axiom into a set of equivalent structural hypersequent rules.

Definition 5.1. A hypersequent $G$ is a multiset $S_{1}|\ldots| S_{n}$ where each $S_{i}$ for $i=1, \ldots, n$ is a sequent, called a component of the hypersequent. A hypersequent is called singleconclusion if all its components are single-conclusion, it is called multiple-conclusion otherwise.

The symbol "|" is intended to denote disjunction at the meta-level. (This will be made precise in Definition 5.2 below.) As sequents are assumed here to be single-conclusion, hypersequents are likewise assumed to be single-conclusion.

Like sequent calculi, hypersequent calculi consist of initial hypersequents, logical rules, cut and structural rules. Initial hypersequents, cut and logical rules are the same as those in sequent calculi, except that a "side hypersequent" may occur, denoted here by the variable $G$. Structural rules are divided into two categories: internal and external rules. The former deal with formulas within sequents as in sequent calculi. External rules manipulate whole sequents. For example, external weakening and contraction rules $(E W)$ and $(E C)$ in Figure 1 add and contract components respectively.

Henceforth we will consider HFLe the hypersequent version of FLe (Figure 1). Let $\vdash_{\text {HFLe }}$ indicate the derivability relation in HFLe. Note that the "hyperlevel" of HFLe is in fact redundant, in the sense that $\vdash_{\text {HFLe }}$ $S_{1}|\cdots| S_{n}$ if and only if for some $i \in\{1, \ldots, n\}$, already $\vdash_{\text {FLe }} S_{i}$.

In hypersequent calculi it is however possible to define additional external structural rules which simultaneously act on several components of one or more hypersequents. It is this type of rule which increases the expressive power of hypersequent calculi compared to ordinary sequent calculi. Examples of these rules are Avron's communication rule $(\mathrm{com})$ and $(l q)$ in Figure 3. E.g., extending the hypersequent version of $\mathbf{L J}$, that is HFLewc, by

- (com) we get a cut-free calculus for Gödel logic, axiomatized by (any Hilbert system for) intuitionistic logic IL + (prelinearity), see [2].

- $(l q)$ we get a cut-free calculus for the intermediate logic $L Q$ axiomatized by IL + (weak excluded middle), see [5].

More formally, by a hypersequent structural rule (hyperstructural rule, for short) we mean $(E W),(E C)$ and any rule, except for (init) and (cut), of the form $(n \geq 0)$

$$
\frac{G\left|\Upsilon_{1}^{\prime} \Rightarrow \Psi_{1}^{\prime} \quad \cdots \quad G\right| \Upsilon_{n}^{\prime} \Rightarrow \Psi_{n}^{\prime}}{G\left|\Upsilon_{1} \Rightarrow \Psi_{1}\right| \cdots \mid \Upsilon_{m} \Rightarrow \Psi_{m}}(h r)
$$

where $G$ is a metavariable that stands for hypersequents, and $\Upsilon_{i}, \Psi_{i}, \Upsilon_{j}^{\prime}, \Upsilon_{j}^{\prime}$ are as in structural rules. Observe that, with the notable exceptions of $(E C)$ and $(E W)$, each premise of a hypersequent structural rule contains exactly one active component $\Upsilon_{i}^{\prime} \Rightarrow \Psi_{i}^{\prime}$.

Two hypersequent rules $\left(h r_{0}\right)$ and $\left(h r_{1}\right)$ are equivalent in HFLe if the derivability relations $\vdash_{\mathbf{H F L e}+\left(h r_{0}\right)}$ and $\vdash_{\text {HFLe }+\left(h r_{1}\right)}$ coincide when restricted to sequents ${ }^{3}$ : $\mathcal{S} \vdash_{\text {HFLe }+\left(h r_{0}\right)} \quad S_{0}$ iff $\mathcal{S} \vdash_{\text {HFLe }+\left(h r_{1}\right)} \quad S_{0}$ for any set $\mathcal{S} \cup\left\{S_{0}\right\}$ of sequents.

We introduce below an algorithm to transform axioms in the subclass $\mathcal{P}_{3}^{\prime}$ of $\mathcal{P}_{3}$ into equivalent hyperstructural rules. Let us begin with establishing a connection between the two derivability relations $\vdash_{\text {FLe }}$ and $\vdash_{\text {HFLe }}$.

Definition 5.2. We define the interpretation function ()$^{I}$ as follows:

1. $\left(\alpha_{1}, \ldots, \alpha_{n} \Rightarrow \beta\right)^{I}=\alpha_{1} \cdots \alpha_{n} \rightarrow \beta$.

2. $\left(\alpha_{1}, \ldots, \alpha_{n} \Rightarrow\right)^{I}=\alpha_{1} \cdots \alpha_{n} \rightarrow 0$.

3. $\left(S_{1}|\cdots| S_{n}\right)^{I}=\left(S_{1}^{I}\right)_{\wedge 1} \vee \cdots \vee\left(S_{n}^{I}\right)_{\wedge 1}$.

Note that our interpretation of "|" differs from that of [11] due to lack of weakening and linearity.

We obviously have $\mathcal{S} \vdash_{\text {FLe }+\psi} \quad S_{0}$ if and only if $\mathcal{S} \vdash_{\text {HFLe }+\psi} S_{0}$ for any axiom $\psi$ and any set $\mathcal{S} \cup\left\{S_{0}\right\}$ of sequents. However, the next proposition gives us a crucial step to "unfold" an axiom $\psi$ to a hyperstructural rule in HFLe.

${ }^{3}$ The reason for this restriction is that we are primarily interested in derivability relations on formulas (or at most sequents); hypersequents are merely a convenient means to obtain analytic calculi. Note that our main theorem in this section (Theorem 5.6) holds only with this restricted notion of equivalence. 


\begin{tabular}{|c|c|c|c|}
\hline$\frac{\Gamma \Rightarrow \Pi}{\Delta, \Gamma \Rightarrow \Pi}(w)$ & $\frac{\Gamma \Rightarrow}{\Gamma \Rightarrow \Pi}\left(w^{\prime}\right)$ & $\frac{\Delta, \Delta, \Gamma \Rightarrow \Pi}{\Delta, \Gamma \Rightarrow \Pi}(c)$ & $\frac{\Delta_{1}, \Gamma \Rightarrow \Pi \quad \Delta_{2}, \Gamma \Rightarrow \Pi}{\Delta_{1}, \Delta_{2}, \Gamma \Rightarrow \Pi}$ (mingle) \\
\hline$\frac{\left\{\Delta_{i_{1}}, \ldots, \Delta_{i_{m}}, \Gamma=\right.}{\Delta_{1}, \ldots, \Delta}$ & $\frac{\Pi\}_{i_{1}, \ldots, i_{m} \in\{1, \ldots, n\}}}{{ }_{n}, \Gamma \Rightarrow \Pi}$ & $\left(k^{\}} \operatorname{lot}_{m}^{n}\right) \frac{G \mid \Gamma, \Delta \Rightarrow \Pi}{G|\Gamma \Rightarrow| \Delta \Rightarrow \Pi}(\mathrm{em})$ & $\frac{\left\{G \mid \Gamma_{i}, \Gamma_{j} \Rightarrow \Pi_{i}\right\}_{0 \leq i, j \leq k, i \neq j}}{G\left|\Gamma_{0} \Rightarrow \Pi_{0}\right| \ldots \mid \Gamma_{k} \Rightarrow \Pi_{k}}(B w k)$ \\
\hline$\frac{G \mid \Gamma, \Delta \Rightarrow}{G|\Gamma \Rightarrow| \Delta \Rightarrow}(l q)$ & $\frac{G \mid \Delta_{1}, \Gamma_{1} \Rightarrow \Pi_{1}}{G \mid \Delta_{2}, \Gamma_{1} \Rightarrow 1}$ & $\frac{G \mid \Delta_{2}, \Gamma_{2} \Rightarrow \Pi_{2}}{\Pi_{1} \mid \Delta_{1}, \Gamma_{2} \Rightarrow \Pi_{2}}(\mathrm{com}) \quad \frac{\{G \mid}{G \mid \Gamma_{0}=}$ & $\frac{\left.{ }_{i}, \Gamma_{j} \Rightarrow \Pi_{i}\right\}_{0 \leq i \leq k-1 ; i+1 \leq j \leq k}}{\Pi_{0}|\ldots| \Gamma_{k-1} \Rightarrow \Pi_{k-1} \mid \Gamma_{k} \Rightarrow}(B c k)$ \\
\hline
\end{tabular}

Figure 3. Some Known (Hyper) structural rules

Proposition 5.3. For any hypersequent $G$ and any set $\mathcal{S} \cup$ $\left\{S_{0}\right\}$ of sequents, we have $\{G\} \cup \mathcal{S} \vdash_{\mathrm{HFLe}} S_{0}$ iff $\left\{\Rightarrow G^{I}\right\} \cup$ $\mathcal{S} \vdash_{\text {HFLe }} S_{0}$ iff $\left\{\Rightarrow G^{I}\right\} \cup \mathcal{S} \vdash_{\text {FLe }} S_{0}$.

Proof. $\left\{\Rightarrow G^{I}\right\} \cup \mathcal{S} \vdash_{\text {FLe }} S_{0}$ obviously implies $\left\{\Rightarrow G^{I}\right\} \cup$ $\mathcal{S} \vdash_{\text {HFLe }} S_{0}$, which in turn implies $\{G\} \cup \mathcal{S} \vdash_{\text {HFLe }} S_{0}$ by $G \vdash_{\mathbf{H F L e}} \Rightarrow G^{I}$. For instance when $G=(\Rightarrow \alpha \mid \Rightarrow \beta)$, we have

$$
\frac{\Rightarrow \alpha \mid \Rightarrow \beta \frac{\overline{\Rightarrow 1}(1 r)}{\Rightarrow 1 \mid \Rightarrow \beta}(E W) \quad(\wedge r) \quad \frac{\bar{\Rightarrow}}{\Rightarrow \alpha_{\wedge 1} \mid \Rightarrow 1}(1 r)}{\Rightarrow \alpha_{\wedge 1} \mid \Rightarrow \beta}(\wedge r)
$$

To show that $\{G\} \cup \mathcal{S} \vdash_{\text {HFLe }} S_{0}$ implies $\left\{\Rightarrow G^{I}\right\} \cup \mathcal{S} \vdash_{\text {FLe }}$ $S_{0}$, we prove by induction on the length of derivation that $\{G\} \cup \mathcal{S} \vdash_{\text {HFLe }} G_{0}$ implies $\left\{\Rightarrow G^{I}\right\} \cup \mathcal{S} \vdash_{\text {FLe }} G_{0}^{I}$ for any hypersequent $G_{0}$. The claim then follows since $S_{0}^{I}$ implies $S_{0}$ in FLe.

The base case being easy. For the inductive case it is enough to observe that for each inference rule in HFLe with premises $G\left|S_{1}, \ldots, G\right| S_{n}$ and conclusion $G \mid S_{0}$, the sequent $\left(G \mid S_{1}\right)^{I}, \ldots,\left(G \mid S_{n}\right)^{I} \Rightarrow\left(G \mid S_{0}\right)^{I}$ is provable in FLe. For instance we have for the $\wedge$ right rule: (assume for simplicity that $G$ consists of a single component)

$$
\begin{aligned}
& \vdash_{\mathrm{FLe}}\left(G^{I}\right)_{\wedge 1} \vee(\Gamma \Rightarrow \alpha)_{\wedge 1}^{I},\left(G^{I}\right)_{\wedge 1} \vee(\Gamma \Rightarrow \beta)_{\wedge 1}^{I} \\
& \Rightarrow\left(G^{I}\right)_{\wedge 1} \vee(\Gamma \Rightarrow \alpha \wedge \beta)_{\wedge 1}^{I} .
\end{aligned}
$$

Corollary 5.4. For any hypersequent $G$ and any set $\mathcal{S} \cup\left\{S_{0}\right\}$ of sequents, we have $\mathcal{S} \vdash_{\mathrm{HFLe}+G} S_{0}$ iff $\mathcal{S} \vdash_{\mathrm{HFLe}+\left(\Rightarrow G^{I}\right)} S_{0}$ iff $\mathcal{S} \vdash_{\mathrm{FLe}+\left(\Rightarrow G^{I}\right)} S_{0}$.

The key Lemma 4.1 naturally extends to hypersequents. We state it in a slightly generalized form for later use.

Lemma 5.5. Let $\Phi, \Phi_{1}, \ldots, \Phi_{m}$ be (meta)hypersequents consisting of metavariables. The hypersequent rule

$$
\frac{G\left|\Phi_{1} \quad \cdots \quad G\right| \Phi_{m}}{G|\Phi| \psi_{1}, \ldots, \psi_{n} \Rightarrow \xi}
$$

is equivalent to each of the rules

$\frac{\overrightarrow{G \mid \Phi} \quad G\left|\Upsilon_{1} \Rightarrow \psi_{1} \quad \cdots \quad G\right| \Upsilon_{n} \Rightarrow \psi_{n}}{G|\Phi| \Upsilon_{1}, \ldots, \Upsilon_{n} \Rightarrow \xi} \frac{\overrightarrow{G \mid \Phi} \quad G \mid \xi, \Upsilon \Rightarrow \Psi}{G|\Phi| \psi_{1}, \ldots, \psi_{n}, \Upsilon \Rightarrow \Psi}$ where $\overrightarrow{G \mid \Phi}=\left(G\left|\Phi_{1}, \cdots, G\right| \Phi_{m}\right), \Upsilon_{i}$ is a fresh metavariable $\alpha_{i}$ or $\Gamma_{i}$, and $\Upsilon \Rightarrow \Psi$ is either $\Rightarrow \beta$ or $\Sigma \Rightarrow \Pi$ with $\beta, \Sigma, \Pi$ fresh.

Proof. Proceed as the proof of Lemma 4.1. To see that the third rule implies the first one, instantiate $\Upsilon \Rightarrow \Psi$ with $\Rightarrow \xi$. The converse direction follows by $(c u t)$.

\section{Theorem 5.6.}

1. Every axiom in $\mathcal{P}_{3}^{\prime}$ is equivalent to a finite set of hyperstructural rules in HFLe.

2. Every axiom in $\mathcal{P}_{3}$ is equivalent to a finite set of hyperstructural rules in HFLew.

Proof. 1. By Lemma 3.5, every axiom in $\mathcal{P}_{3}^{\prime}$ is equivalent to a finite set of axioms $\left(\psi_{1}\right)_{\wedge 1} \vee \cdots \vee\left(\psi_{n}\right)_{\wedge 1}$ where $\psi_{1}, \ldots, \psi_{n}$ are $\mathcal{N}_{2}$-normal. By Corollary 5.4, the latter is equivalent to $\Rightarrow \psi_{1}|\cdots| \Rightarrow \psi_{n}$ in HFLe, which is in turn equivalent to $\Phi=\left(G\left|\Rightarrow \psi_{1}\right| \cdots \mid \Rightarrow \psi_{n}\right)$ with $G$ a metavariable for hypersequents, by $(E W)$ and instantiation of $G$ with the empty hypersequent. By applying the procedure described in the proof of Theorem 4.2 to each component of $\Phi$ we obtain a finite set of hyperstructural rules, which is equivalent to $\Phi$ by Lemma 5.5.

2. Follows by $\vdash_{\text {FLew }} \alpha \leftrightarrow \alpha \wedge 1$.

Example 5.7. The $\mathcal{P}_{3}^{\prime}$ version of the weak nilpotent minimum axiom (see Example 3.2), i.e.,

$(\neg(\alpha \cdot \beta))_{\wedge 1} \vee(\alpha \wedge \beta \rightarrow \alpha \cdot \beta)_{\wedge 1}$

is transformed into the hyperstructural rule $\left(w n m_{0}\right)$ as follows:

$$
\begin{array}{cc}
\longrightarrow & \overline{G|\Rightarrow \neg(\alpha \cdot \beta)| \Rightarrow \alpha \wedge \beta \rightarrow \alpha \cdot \beta} \\
\longrightarrow & \frac{G|\alpha, \beta \Rightarrow| \alpha \wedge \beta \Rightarrow \alpha \cdot \beta}{G \Rightarrow \alpha \wedge \beta \quad G \mid \alpha \cdot \beta \Rightarrow \sigma} \\
\longrightarrow \quad & \frac{G|\tau \Rightarrow \alpha \quad G| \tau \Rightarrow \beta \quad G \mid \alpha, \beta \Rightarrow \sigma}{G|\alpha, \beta \Rightarrow| \tau \Rightarrow \sigma} \\
& \frac{\left.G n m_{0}\right)}{G|\alpha, \beta \Rightarrow| \tau \Rightarrow \sigma}
\end{array}
$$


Linearity and the $\mathcal{P}_{3}^{\prime}$ version of the weak law of excluded middle (see Fig. 2) are transformed into

$\frac{G|\beta \Rightarrow \gamma \quad G| \alpha \Rightarrow \delta}{G|\alpha \Rightarrow \gamma| \beta \Rightarrow \delta}\left(\operatorname{com}_{0}\right) \quad \frac{G|\gamma \Rightarrow \alpha \quad G| \beta, \alpha \Rightarrow}{G|\gamma \Rightarrow| \beta \Rightarrow}\left(l q_{0}\right)$

\section{Rule Completion}

As seen in the previous section, all axioms in $\mathcal{P}_{3}^{\prime}$ (and in presence of weakening in $\mathcal{P}_{3}$ ) can be transformed into hyperstructural rules. These rules however do not always preserve cut admissibility once added to HFLe. For instance, HFLewc extended with

$$
\frac{G \mid \Gamma, \Delta \Rightarrow \alpha}{G|\Gamma \Rightarrow \alpha| \Delta \Rightarrow \alpha}\left(S_{I}\right)
$$

does not enjoy cut admissibility, see [3]. Nevertheless, the above rule can be transformed into an equivalent one (in HFLe) which does preserve cut admissibility. The same applies to any hyperstructural rule when we admit weakening (see Section 7), or when the rule satisfies the acyclicity condition below. The purpose of this section is to describe this transformation (we refer to it as completion), which extends a similar procedure in [18] that works for suitable sequent structural rules in $\mathbf{F L}$, and is analogous to the principle of reflection in [17].

Definition 6.1. Given a hyperstructural rule

$$
\frac{G\left|\Upsilon_{1}^{\prime} \Rightarrow \Psi_{1}^{\prime} \quad \cdots \quad G\right| \Upsilon_{n}^{\prime} \Rightarrow \Psi_{n}^{\prime}}{G\left|\Upsilon_{1} \Rightarrow \Psi_{1}\right| \cdots \mid \Upsilon_{m} \Rightarrow \Psi_{m}}(h r)
$$

we build its dependency graph $D(h r)$ as follows:

- The vertices of $D(h r)$ are the metavariables for formulas occurring in the premises $G \mid \Upsilon_{1}^{\prime} \Rightarrow$ $\Psi_{1}^{\prime}, \cdots, G \mid \Upsilon_{n}^{\prime} \Rightarrow \Psi_{n}^{\prime}$ (we do not distinguish occurrences).

- There is a directed edge $\alpha \longrightarrow \beta$ in $D(r)$ if and only if there is a premise $G \mid \Upsilon_{i}^{\prime} \Rightarrow \Psi_{i}^{\prime}$ such that $\alpha$ occurs in $\Upsilon_{i}^{\prime}$ and $\beta=\Psi^{\prime}$.

A hyperstructural rule $(h r)$ is said to be acyclic if $D(h r)$ is acyclic.

Example 6.2. The rules $\left(w n m_{0}\right),\left(c o m_{0}\right)$ and $\left(l q_{0}\right)$ in Example 5.7 and $\left(S_{I}\right)$ above are acyclic, while this is not the case for the rule

$$
\frac{G|\gamma, \alpha \Rightarrow \beta \quad G| \beta \Rightarrow \alpha}{G \mid \gamma \Rightarrow \beta}
$$

1. Preliminary step. Given any hyperstructural rule, we replace each metavariable $\Gamma$ for multisets of formulas (resp. each metavariable $\Pi$ for stoups), if any, by a fresh metavariable $\beta_{\Gamma}$ (resp. $\beta_{\Pi}$ ) for formulas.
Clearly the original rule implies the new one. The converse also holds because any multiset $\Gamma=\alpha_{1}, \ldots, \alpha_{n}$ of formulas (resp. the empty stoup $\Pi=\emptyset$ ) can be turned into a single formula $\circ \Gamma=\alpha_{1} \cdots \alpha_{n}$ (resp. 0 ). Hence given concrete instances of the premises of the original rule, one can first replace a multiset $\Gamma$ (resp. the empty stoup) with formula $\circ \Gamma$ (resp. 0 ), apply the new rule and later on recover the multiset $\Gamma$ (resp. the empty stoup) by the invertibility of $(\cdot l),(1 l)$ and $(0 r)$.

This step is not needed when the given hyperstructural rule contains neither $\Gamma$ nor $\Pi$, as in the case of the rules generated by the algorithms in Theorems 4.2 and 5.6. Notice that this step preserves acyclicity, i.e. if the original rule is acyclic, so is the rule after applying the preliminary step.

Example 6.3. Applied to the rule $\left(S_{I}\right)$ above the preliminary yields

$$
\frac{G \mid \beta_{\Gamma}, \beta_{\Delta} \Rightarrow \alpha}{G\left|\beta_{\Gamma} \Rightarrow \alpha\right| \beta_{\Delta} \Rightarrow \alpha}\left(S^{\prime}\right)
$$

2. Restructuring. Given any hyperstructural rule only containing metavariables for formulas. We replace each component $\left(\alpha_{1}, \ldots, \alpha_{n} \Rightarrow \beta\right)$ in its conclusion with $\left(\Gamma_{1}, \ldots, \Gamma_{n}, \Sigma_{\beta} \Rightarrow \Pi_{\beta}\right)$ and add $n+1$ premises $\left(G \mid \Gamma_{1} \Rightarrow\right.$ $\left.\alpha_{1}\right), \ldots,\left(G \mid \Gamma_{n} \Rightarrow \alpha_{n}\right),\left(G \mid \beta, \Sigma_{\beta} \Rightarrow \Pi_{\beta}\right)$, where $\Gamma_{1}, \ldots, \Gamma_{n}, \Sigma_{\beta}, \Pi_{\beta}$ are fresh and mutually distinct metavariables. Likewise, we replace each component in its conclusion of the form $\left(\alpha_{1}, \ldots, \alpha_{n} \Rightarrow\right)$ with $\left(\Gamma_{1}, \ldots, \Gamma_{n} \Rightarrow\right)$ and add $n$ premises $\left(G \mid \Gamma_{1} \Rightarrow \alpha_{1}\right)$, $\ldots,\left(G \mid \Gamma_{n} \Rightarrow \alpha_{n}\right)$. As a result, we obtain a new rule in which

(linear-conclusion) each metavariable occurs (at most) once in the conclusion

(separation) no metavariable occurring on the LHS (resp. RHS) of a component of the conclusion does occur on the RHS (resp. LHS) of a premise, and

(coupling) any pair $\left(\Sigma_{\beta}, \Pi_{\beta}\right)$ of metavariables associated to the same occurrence of $\beta$ always occur together, namely $\Sigma_{\beta}$ occurs in a premise iff $\Pi_{\beta}$ does.

Example 6.4. Applied to $\left(w_{n} m_{0}\right)$ in Example 5.7, the restructuring step yields a new rule $\left(\right.$ wn $\left._{1}\right)$

$$
\begin{array}{cccc}
G \mid \tau \Rightarrow \alpha & G \mid \tau \Rightarrow \beta & G \mid \alpha, \beta \Rightarrow \sigma \\
G \mid \Gamma \Rightarrow \alpha & G \mid \Delta \Rightarrow \beta & G \mid \Lambda \Rightarrow \tau & G \mid \sigma, \Sigma \Rightarrow \Pi \\
\hline & G|\Gamma, \Delta \Rightarrow| \Lambda, \Sigma \Rightarrow \Pi
\end{array}
$$

while applied to the rule $\left(S^{\prime}\right)$ in Example 6.3 this step yields

$$
\begin{gathered}
G\left|\beta_{\Gamma}, \beta_{\Delta} \Rightarrow \alpha \quad G\right| \Gamma_{1} \Rightarrow \beta_{\Gamma} \\
G\left|\alpha, \Lambda_{1} \Rightarrow \Pi_{1} \quad G\right| \Gamma_{2} \Rightarrow \beta_{\Delta} \quad G \mid \alpha, \Lambda_{2} \Rightarrow \Pi_{2}
\end{gathered}
$$


When the original rule is acyclic, so is the resulting rule as we add only metavariables for multisets and stoups. The equivalence with the original rule is ensured by Lemma 5.5 .

3. Cutting. Given any acyclic hyperstructural rule. We eliminate from the set $\mathcal{G}$ of its premises all metavariables not occurring in the conclusion (we call these variables redundant). The procedure is as follows.

Let $\alpha$ be such a redundant metavariable and $\mathcal{G}_{1}=$ $\left\{G \mid \Upsilon_{i}^{\prime} \Rightarrow \alpha: 1 \leq i \leq k\right\}$ be the subset of premises which have $\alpha$ on the RHS, and $\mathcal{G}_{2}=\left\{G \mid \Upsilon_{j}, \alpha, \ldots, \alpha \Rightarrow \Psi_{j}\right.$ : $1 \leq j \leq m\}$ be the set of those which have one or more occurrences of $\alpha$ on the LHS, where $\Upsilon_{j}$ does not contain $\alpha$. By acyclicity, $\alpha$ does not appear in $\Upsilon_{i}^{\prime}$ and $\Psi_{j}$.

If $k=0$ (resp. $m=0$ ), then remove $\mathcal{G}_{2}$ (resp. $\mathcal{G}_{1}$ ) from $\mathcal{G}$. The resulting rule implies the original one, by instantiating $\alpha$ with $\perp$ (resp. $\top$ ). (This remains true even if $\perp, \top$ are not in the language.)

Otherwise, let $\mathcal{G}_{3}$ be the set of all hypersequents of the form $G \mid \Upsilon_{j}, \Upsilon_{i_{1}}^{\prime}, \ldots, \Upsilon_{i_{p}}^{\prime} \Rightarrow \Psi_{j}$, where $1 \leq j \leq m$ and $1 \leq i_{1}, \ldots, i_{p} \leq k$. We replace $\mathcal{G}_{1} \cup \mathcal{G}_{2}$ with $\mathcal{G}_{3}$ thus obtaining a new hyperstructural rule. It is clear that the acyclicity is preserved by this transformation and the number of redundant variables decreases by one. Hence by repeating this process, we obtain a hyperstructural rule without redundant variables.

Example 6.5. Applied to the metavariables $\tau$ and $\sigma$ of the rule $\left(w n m_{1}\right)$ in the previous example, the cutting step yields

$$
\begin{aligned}
& G|\Lambda \Rightarrow \alpha \quad G| \Lambda \Rightarrow \beta \\
& G|\Gamma \Rightarrow \alpha \quad G| \Delta \Rightarrow \beta \quad G \mid \alpha, \beta, \Sigma \Rightarrow \Pi \\
& G|\Gamma, \Delta \Rightarrow| \Lambda, \Sigma \Rightarrow \Pi
\end{aligned}
$$

and applied further on $\alpha$ and $\beta$,

$$
\begin{gathered}
G|\Gamma, \Delta, \Sigma \Rightarrow \Pi, \quad G| \Lambda, \Delta, \Sigma \Rightarrow \Pi \\
G|\Gamma, \Lambda, \Sigma \Rightarrow \Pi, \quad G| \Lambda, \Lambda, \Sigma \Rightarrow \Pi \\
\hline G|\Gamma, \Delta \Rightarrow| \Lambda, \Sigma \Rightarrow \Pi
\end{gathered}
$$

To see that this step preserves equivalence, we show that the two rules above $\left(\left(w n m_{2}\right)\right.$ and $\left.(w n m)\right)$ are equivalent. It is clear that the conclusion $G|\Gamma, \Delta \Rightarrow| \Lambda, \Sigma \Rightarrow \Pi$ is derivable from the premises of $\left(w n m_{2}\right)$ by using $(c u t)$ and $(w n m)$. Conversely, consider concrete instances of the premises of $(w n m)$. Let $\circ \Lambda$ be the fusion of all formulas in $\Lambda$, and $\bar{\alpha}=\circ \Lambda \vee \circ \Gamma, \bar{\beta}=\circ \Lambda \vee \circ \Delta$. Since $(G \mid \Lambda \Rightarrow \bar{\alpha})$, $(G \mid \Lambda \Rightarrow \bar{\beta}),(G \mid \Gamma \Rightarrow \bar{\alpha})$ and $(G \mid \Delta \Rightarrow \bar{\beta})$ are provable and $G \mid \bar{\alpha}, \bar{\beta}, \Sigma \Rightarrow \Pi$ is derivable from the premises of $(w n m)$, we obtain the conclusion by $\left(w_{n n}\right)$.

We call completed any hyperstructural rule obtained by applying the above completion procedure (steps 1-3). Any completed hyperstructural rule satisfies the properties (linear-conclusion) and (coupling) together with a strengthened form of (separation):

(strong subformula property) every metavariable occurring on the LHS (resp. RHS) in a premise also occurs on the LHS (resp. RHS) of the conclusion.

Example 6.6. The completion of the rule $\left(S_{I}\right)$ leads to the rule

$$
\frac{G\left|\Gamma_{1}, \Gamma_{2}, \Lambda_{1} \Rightarrow \Pi_{1} \quad G\right| \Gamma_{1}, \Gamma_{2}, \Lambda_{2} \Rightarrow \Pi_{2}}{G\left|\Gamma_{1}, \Lambda_{1} \Rightarrow \Pi_{1}\right| \Gamma_{2}, \Lambda_{2} \Rightarrow \Pi_{2}}
$$

suggested by Mints (see [3]) and equivalent to (com) in presence of weakening and contraction.

Example 6.7. By applying to the axioms in Figure 2 the translation of Sections 4 and 5 followed by the completion procedure, we obtain the known rules in Figure 3 (up to contraction $(c)$ for $(B w k)$ and $(B c k))$.

\section{On the power of (hyper)structural rules}

If we admit weakening the completion procedure described in Section 6 does not need the acyclicity condition anymore and hence all (hyper)structural rule can be completed. This observation leads to two results (Corollary 7.2 and Corollary 7.3) that shed light on the expressive power of single-conclusion (hyper)sequent calculi.

\section{Theorem 7.1.}

(a) Any acyclic hyperstructural rule can be transformed into a completed rule which is equivalent in HFLe;

(b) Any hyperstructural rule can be transformed into a completed rule which is equivalent in HFLew.

Proof. (a) Follows by results in the preceeding section.

(b) Steps 1 and 2 in Section 6 can be applied to any hyperstructural rule. As for step 3 (Cutting), all premises in $\mathcal{G}_{i}, i=1,2,3$ of the form $\Upsilon, \alpha \Rightarrow \alpha$ can be simply removed, being already derivable by weakening in HFLew. It is easy to see that the resulting rule is equivalent to the original rule in HFLew.

The completion procedure for hyperstructural rules outlined in Section 6 subsumes completion of structural rules in sequent calculi. Hence

Corollary 7.2. Any structural rule is either derivable in Gentzen's LJ or derives every formula in $\mathbf{L J}$.

Proof. Given a structural rule $(r)$, we apply the completion procedure to obtain, by Theorem 7.1(b), a completed rule $\left(r^{\prime}\right)$ equivalent to $(r)$ in LJ. If $\left(r^{\prime}\right)$ has no premises, by linear-conclusion any formula is provable in $\mathbf{L J}+\left(r^{\prime}\right)$ and hence in $\mathbf{L J}+(r)$. Otherwise, the conclusion of $\left(r^{\prime}\right)$ is derivable from any of its premises by weakening and contraction due to the strong subformula property. 
Let HSM be the hypersequent calculus HFLewc + $(\mathrm{com})+(B c 2)$ (see Figure 3) introduced in [5] for threevalued Gödel logic SM - the strongest intermediate logic, semantically characterized by linearly ordered Kripke models containing two worlds.

Corollary 7.3. Any hyperstructural rule is either derivable in HSM or derives $\alpha \vee \neg \alpha^{n}$ in HFLew, for some $n \in N$.

Proof. First note that both rules

$$
\frac{G\left|\Upsilon_{1}, \Upsilon_{2} \Rightarrow \Pi_{1} \quad G\right| \Upsilon_{0}, \Upsilon_{2} \Rightarrow \Pi_{2}}{G\left|\Upsilon_{1} \Rightarrow \Pi_{1}\right| \Upsilon_{2} \Rightarrow \Pi_{2} \mid \Upsilon_{0} \Rightarrow}\left(B c 2^{\prime}\right)
$$

and

$\frac{G \mid \Upsilon_{1}, \ldots, \Upsilon_{n} \Rightarrow \Pi_{1} \quad\left\{G \mid \Upsilon_{0}, \Upsilon_{1}, \ldots, \Upsilon_{n} \Rightarrow \Pi_{i}\right\}_{2 \leq i \leq n}}{G\left|\Upsilon_{1} \Rightarrow \Pi_{1}\right| \cdots\left|\Upsilon_{n} \Rightarrow \Pi_{n}\right| \Upsilon_{0} \Rightarrow}$ $\left(B c n^{\prime}\right)$

are derivable in HSM. Indeed $\left(B c 2^{\prime}\right)$ follows by $(\mathrm{com}),(B c 2),(c),(E W)$ and $(E C)$, while $\left(B c n^{\prime}\right)$ is derivable in HSM by $n-1$ applications of both $(w)$ and $\left(B c 2^{\prime}\right)$ with premises $G \mid \Upsilon_{1}, \ldots, \Upsilon_{n} \Rightarrow \Pi_{1}$ and $G \mid \Upsilon_{0}, \ldots, \Upsilon_{n} \Rightarrow \Pi_{i}(2 \leq i \leq n)$, followed by several applications of $(\mathrm{com}),(\mathrm{c}),(E W)$ and $(E C)$.

Given any (hyper)structural rule. By Theorem 7.1(b) it is equivalent in HFLew to a completed rule, say $(h r)$. If at least one active component in the premises of $(h r)$ has empty RHS then $(h r)$ is derivable in HSM (use $(c o m),(w),\left(w^{\prime}\right),(c)$ and $\left.(E W)\right)$. Otherwise, we can assume that $(h r)$ has the form

$$
\frac{G\left|\Xi_{1} \Rightarrow \Pi_{1}^{\prime} \quad \ldots \quad G\right| \Xi_{k} \Rightarrow \Pi_{k}^{\prime}}{G\left|\Upsilon_{1} \Rightarrow \Pi_{1}\right| \cdots\left|\Upsilon_{n} \Rightarrow \Pi_{n}\right| \Upsilon_{1}^{\prime} \Rightarrow|\ldots| \Upsilon_{m}^{\prime} \Rightarrow}
$$

with $m \geq 0$. We can also assume $\Pi_{1}, \ldots, \Pi_{n} \subseteq$ $\left\{\Pi_{1}^{\prime}, \ldots, \Pi_{k}^{\prime}\right\}$ as if e.g. $\Pi_{1} \notin\left\{\Pi_{1}^{\prime}, \ldots, \Pi_{k}^{\prime}\right\}$ then $(h r)$ is equivalent in HFLew to the rule with premises $G \mid \Xi_{1} \Rightarrow$ $\Pi_{1}^{\prime}, \ldots, G \mid \Xi_{k} \Rightarrow \Pi_{k}^{\prime}$ and conclusion $G \mid \Upsilon_{2} \Rightarrow$ $\Pi_{2}|\ldots| \Upsilon_{n} \Rightarrow \Pi_{n}\left|\Upsilon_{1}^{\prime} \Rightarrow\right| \ldots\left|\Upsilon_{m}^{\prime} \Rightarrow\right| \Upsilon_{1} \Rightarrow$. Let $\Upsilon_{0}=\Upsilon_{1}^{\prime}, \ldots, \Upsilon_{m}^{\prime}$ and consider the rule $(h r)^{\prime}$

$$
\frac{G\left|\Xi_{1} \Rightarrow \Pi_{1}^{\prime} \quad \ldots \quad G\right| \Xi_{k} \Rightarrow \Pi_{k}^{\prime}}{G\left|\Upsilon_{1} \Rightarrow \Pi_{1}\right| \cdots\left|\Upsilon_{n} \Rightarrow \Pi_{n}\right| \Upsilon_{0} \Rightarrow}
$$

obtained applying to the conclusion of $(h r):(E W)$, when $m=0$ and both $(w)$ and $(E C)$, when $m>1$. Note that $(h r)^{\prime}$ is derivable from $(h r)$ in HFLew and satisfies linearconclusion. Two cases can occur:

1. There is a premise $G \mid \Xi_{j} \Rightarrow \Pi_{j}^{\prime}$ in $(h r)^{\prime}$ such that $\Xi_{j} \cap \Upsilon_{0}=\emptyset$. As $\Pi_{1}, \ldots, \Pi_{n} \subseteq\left\{\Pi_{1}^{\prime}, \ldots, \Pi_{k}^{\prime}\right\}$, the conclusion of $(h r)^{\prime}$ is derivable from (some of) its premises using $\left(B c n^{\prime}\right)$ and $(w)$, from which the conclusion of $(h r)$ follows by several applications of $(\mathrm{com})$ and $(c)$. Hence $(h r)$ is derivable in HSM.

2. Assume otherwise that each premise $G \mid \Xi_{i} \Rightarrow \Pi_{i}^{\prime}$ of $(h r)^{\prime}$ involves a metavariable in $\Upsilon_{0}$. We instantiate all metavariables in $\Upsilon_{0}$ and those for stoups with $\alpha$, and all others with the empty multiset. Then all the premises of $(h r)^{\prime}$ are of the form $\alpha, \ldots, \alpha \Rightarrow \alpha$ and hence are provable in HFLew. By the linear-conclusion property, the conclusion is of the form $\Rightarrow \alpha|\cdots| \Rightarrow \alpha \mid \alpha, \ldots, \alpha \Rightarrow$, from which $\alpha \vee \neg \alpha^{n}$ is easily derivable in HFLew.

Corollaries 7.2 and 7.3 can be used to establish negative results on the transformation of axioms into inference rules.

Example 7.4. No (hyper)structural rule is equivalent to $((\alpha \rightarrow \beta) \rightarrow \beta) \rightarrow((\beta \rightarrow \alpha) \rightarrow \alpha)$ (Eukasiewicz axiom, see Example 3.2). Indeed this axiom is not valid in SM (take an evaluation $v$ in $\mathbf{S M}$, i.e., $v$ : $\mathbf{S M}$-formulas $\rightarrow\{0,1 / 2,1\}$ with $v(\alpha \rightarrow \beta)=1$ if $v(\alpha) \leq v(\beta)$ and $v(\alpha \rightarrow \beta)=v(\beta)$ otherwise, and assign $v(\alpha)=1 / 2$ and $v(\beta)=0$ ). Moreover for no finite $n$, is $\alpha \vee \neg \alpha^{n}$ derivable from HFLew extended with Łukasiewicz axiom. This follows from the fact that Corollary 5.4 also holds for HFLew and FLew and from the non validity of $\alpha \vee \neg \alpha^{n}$ in infinite-valued Łukasiewicz logic $\mathbf{L}$, which is obtained by adding Łukasiewicz axiom to FLew (take an evaluation $v$ in $\mathbf{L}$, i.e., $v: \mathbf{L}$-formulas $\rightarrow[0,1]$ with $v(\neg \alpha)=1-v(\alpha), v(\alpha \cdot \beta)=\max \{0, v(\alpha)+v(\beta)-1\}$ and assign $v(\alpha)=n /(n+1))$.

\section{Cut Elimination}

We introduce a uniform (and first semantic) proof of cutadmissibility for any (hyper)sequent calculus defined by extending HFLe with any set of completed rules. Our result is obtained by extending to hypersequents a powerful semantic technique introduced by Okada (see e.g. [13]) which proved cut-elimination for (higher order) linear, intuitionistic and classical logics.

Traditional (syntactic) proofs of cut-elimination start with derivations containing cuts and generate derivations without cuts. Semantic proofs go instead in the opposite direction; they start with a notion of cut-free provability and build a model in which cuts are valid.

The latter step is analogous to the process of obtaining the field of reals from the field of rationals $\mathbf{Q}=$ $(Q,+, \cdot, 0,1)$ via the Dedekind-MacNeille completion. For any $X \subseteq Q$, define:

$$
\begin{aligned}
& X^{\triangleright}=\{y: \forall x \in X . x \leq y\} \\
& X^{\triangleleft}=\{y: \forall x \in X . y \leq x\}
\end{aligned}
$$

Then the set $\bar{R}=\left\{X \subseteq Q: X=X^{\triangleright \triangleleft}\right\}$ can be thought of as the set of real numbers extended with $\pm \infty$ and ordered by inclusion $\subseteq$, and one can naturally embed $\mathbf{Q}$ into $\overline{\mathbf{R}}=(\bar{R},+, \cdot, \mathbf{0}, \mathbf{1})$, where $+, \cdot, \mathbf{0}, \mathbf{1}$ are suitably defined, by mapping $r \in Q$ to $r^{\bullet}=r^{\triangleright \triangleleft}=r^{\triangleleft}$. 
This construction yields a continuous structure out of a discontinuous one. In our case, we start with an 'intransitive' structure (as $\Rightarrow$ of a sequent is intransitive in the absence of the cut rule), and obtain a 'transitive' one in which the cut rule is valid. We refer to [15] for further algebraic account.

Let $R$ be a set of completed hyperstructural rules. We write $\vdash_{\mathrm{HFLe}+R}^{c f} G$ if $G$ is cut-free provable in HFLe + $R$. For a set $\mathcal{G}$ of hypersequents, we write $\vdash_{\text {HFLe }+R}^{c f} \mathcal{G}$ if $\vdash_{\text {HFLe }+R}^{c f} G$ for every $G \in \mathcal{G}$.

We denote the set of multisets of formulas by $\mathcal{M}$, the set of sequents by $\mathcal{S}$ and the set of hypersequents by $\mathcal{H}$. We write $\mathcal{M H}$ and $\mathcal{S H}$ for $\mathcal{M} \times \mathcal{H}$ and $\mathcal{S} \times \mathcal{H}$, respectively. The empty hypersequent in $\mathcal{H}$ and the empty multiset in $\mathcal{M}$ are respectively denoted by $\emptyset$ and $\varepsilon$. Given $(\Gamma ; G),(\Delta ; H) \in$ $\mathcal{M H}$ and $(\Sigma \Rightarrow \Pi ; F) \in \mathcal{S H}$, we define:

$$
\begin{array}{rlrl}
(\Gamma ; G) \circ(\Delta ; H) & =(\Gamma, \Delta ; G \mid H) & & \in \mathcal{M H} \\
(\Gamma ; G) @(\Sigma \Rightarrow \Pi ; F) & =\Gamma, \Sigma \Rightarrow \Pi|G| F & \in \mathcal{H}
\end{array}
$$

Then $(\mathcal{M H}, \circ,(\varepsilon ; \emptyset))$ forms a commutative monoid. In the sequel, we write $\mathcal{X}, \mathcal{Y}, \mathcal{Z} \ldots$ for subsets of $\mathcal{M H}$, and $\mathcal{U}, \mathcal{V}, \ldots$ for those of $\mathcal{S H}$. The binary operations $\circ$, @ and | are naturally extended: $\mathcal{X} \circ \mathcal{Y}=\{x \circ y: x \in \mathcal{X}, y \in \mathcal{Y}\}$ and similarly for $\mathcal{X} @ \mathcal{U}$ and $\mathcal{G} \mid \mathcal{G}^{\prime}$.

Furthermore, we define:

$$
\begin{aligned}
& \mathcal{X}^{\triangleright}=\left\{u \in \mathcal{S H}: \forall x \in \mathcal{X} . \vdash_{\mathbf{H F L e}+R}^{c f} x @ u\right\} \\
& \mathcal{U}^{\triangleleft}=\left\{x \in \mathcal{M H}: \forall u \in \mathcal{U} . \vdash_{\text {HFLe }+R}^{c f} x @ u\right\}
\end{aligned}
$$

Notice in particular that if $(\Gamma ; G) \in \mathcal{X}$ (resp. $\in \mathcal{U}^{\triangleleft}$ ) and $(\Delta \Rightarrow \Pi ; H) \in \mathcal{X}^{\triangleright}$ (resp. $\left.\in \mathcal{U}\right)$, the hypersequent $\Gamma, \Delta \Rightarrow$ $\Pi|G| H$ is cut-free provable in HFLe $+R$.

The two operations ()$^{\triangleright}$ and ()$^{\triangleleft}$ form a so-called Galois connection between $\mathcal{P}(\mathcal{M H})$ and $\mathcal{P}(\mathcal{S H}): \mathcal{X} \subseteq$ $\mathcal{U}^{\triangleleft} \Longleftrightarrow \mathcal{U} \subseteq \mathcal{X}^{\triangleright}$, inducing a closure operator ()$^{\triangleright \triangleleft}$ on $\mathcal{P}(\mathcal{M H})$. We have

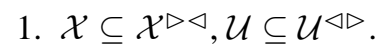

2. $\mathcal{X} \subseteq \mathcal{Y} \Longrightarrow \mathcal{Y}^{\triangleright} \subseteq \mathcal{X}^{\triangleright}, \mathcal{U} \subseteq \mathcal{V} \Longrightarrow \mathcal{V}^{\triangleleft} \subseteq \mathcal{U}^{\triangleleft}$.

3. $\mathcal{X}^{\triangleright \triangleleft \triangleright}=\mathcal{X}^{\triangleright}, \mathcal{U}^{\triangleleft \triangleright \triangleleft}=\mathcal{U}^{\triangleleft}$.

4. $\mathcal{X}^{\triangleright \triangleleft} \circ \mathcal{Y}^{\triangleright \triangleleft} \subseteq(\mathcal{X} \circ \mathcal{Y})^{\triangleright \triangleleft}$.

The last property makes ()$^{\triangleright \triangleleft}$ a nucleus [8]. Let us denote by $\mathcal{C}$ the set of all closed sets w. r. t. ( $)^{\triangleright \triangleleft}$ and define

$$
\begin{aligned}
\mathcal{X} \oplus \mathcal{Y} & =(\mathcal{X} \cup \mathcal{Y})^{\triangleright \triangleleft}, \quad \mathbf{0}=\{(\Rightarrow ; \emptyset)\} \\
\mathcal{X} \otimes \mathcal{Y} & =(\mathcal{X} \circ \mathcal{Y})^{\triangleright \triangleleft}, \quad \mathbf{1}=\{(\epsilon ; \emptyset)\} \triangleright \triangleleft, \\
\mathcal{X} \multimap \mathcal{Y} & =\{y \in \mathcal{M H}: \forall x \in \mathcal{X} . x \circ y \in \mathcal{Y}\}
\end{aligned}
$$

Lemma 8.1 ([14]). $\mathbf{A}=(\mathcal{C}, \cap, \oplus, \otimes,-, \mathcal{M H}, \emptyset \triangleright \triangleleft, \mathbf{1}, \mathbf{0})$ is a bounded pointed commutative residuated lattice. Namely,
- $(\mathcal{C}, \cap, \oplus, \mathcal{M H}, \emptyset \triangleright \triangleleft)$ is a lattice with greatest element $\mathcal{M H}$ and least element $\emptyset \triangleright \triangleleft$;

- $(\mathcal{C}, \otimes, \mathbf{1})$ is a commutative monoid;

- for any $\mathcal{X}, \mathcal{Y}, \mathcal{Z} \in \mathcal{C}, \mathcal{X} \otimes \mathcal{Y} \subseteq \mathcal{Z} \Longleftrightarrow \mathcal{X} \subseteq \mathcal{Y} \multimap \mathcal{Z}$.

0 is just a point and there is no condition on it. Bounded pointed commutative residuated lattices, also known as $F L_{e}$-algebras, give rise to an algebraic semantics for FLe (see [8]). Hence we can interpret our formulas in $\mathbf{A}$.

A valuation on $\mathbf{A}$ is a function ()$^{\bullet}$ that maps each propositional variable $p$ to a closed set $p^{\bullet} \in \mathcal{C}$. It can be naturally extended to arbitrary formulas. If $\Gamma=\alpha_{1}, \ldots, \alpha_{n}$ (resp. $\Pi=\beta$ ), then $\Gamma^{\bullet}=\alpha_{1}^{\bullet} \circ \cdots \circ \alpha_{n}^{\bullet}\left(\right.$ resp. $\left.\Pi^{\bullet}=\beta^{\bullet}\right)$. If $\Gamma$ (resp. $\Pi$ ) is empty, then $\Gamma^{\bullet}=\mathbf{1}$ (resp. $\Pi^{\bullet}=\mathbf{0}$ ). We interpret a sequent $S=\Gamma \Rightarrow \Pi$ by $S^{\bullet}=\Gamma^{\bullet} @\left(\Pi^{\bullet \triangleright}\right)$, and a hypersequent $G=S_{1}|\cdots| S_{n}$ by $G^{\bullet}=S_{1}^{\bullet}|\cdots| S_{n}^{\bullet}$. If $\Gamma$ is empty, then $G^{\bullet}=\{\emptyset\}$. Notice that $S^{\bullet}$ and $G^{\bullet}$ are subsets of $\mathcal{H}$.

Our model supports 'focusing' of a component in a hypersequent:

Lemma 8.2. $\vdash_{\mathrm{HFLe}+R}^{c f}(\Gamma \Rightarrow \Pi \mid G)^{\bullet}$ if and only if $\left(\epsilon ; G^{\bullet}\right) \subseteq \Gamma^{\bullet} \multimap \Pi^{\bullet}$, where $\left(\epsilon ; G^{\bullet}\right)$ denotes the set $\{(\epsilon ; H)$ : $\left.H \in G^{\bullet}\right\}$. In particular when $G$ is empty, we have $\vdash_{\text {HFLe }+R}^{c f}(\Gamma \Rightarrow \Pi)^{\bullet}$ if and only if $(\epsilon ; \emptyset) \in \Gamma^{\bullet} \multimap \Pi^{\bullet}$.

Proof. $(\Rightarrow)$ Let $(\epsilon ; H) \in\left(\epsilon ; G^{\bullet}\right), x \in \Gamma^{\bullet}$ and $u \in \Pi^{\bullet \triangleright}$. Then $x @ u \mid H$ belongs to $(\Gamma \Rightarrow \Pi \mid G)^{\bullet}$, and so is cutfree provable. Since $x @ u \mid H=((\epsilon ; H) \circ x) @ u$, we have $(\epsilon ; H) \circ x \in \Pi^{\bullet \triangleright \triangleleft}=\Pi^{\bullet}$, and hence $(\epsilon ; H) \in \Gamma^{\bullet} \multimap \Pi^{\bullet}$. The converse direction is also easy.

Theorem 8.3 (Soundness). Let ()$^{\bullet}$ be a valuation on $\mathbf{A}$. For any hypersequent $G, \vdash_{\text {HFLe }+R} G$ implies $\vdash_{\text {HFLe }+R}^{c f}$ $G^{\bullet}$. Hence $\vdash_{\mathbf{H F L e}+R} \Gamma \Rightarrow \Pi$ implies $(\epsilon ; \emptyset) \in \Gamma^{\bullet} \underset{\multimap}{\circ} \Pi^{\bullet}$.

Proof. By induction on the length of derivation. The identity axiom, cut and logical rules are dealt with by Lemmas 8.1 and 8.2. For instance, when the derivation ends with an instance of (cut):

$$
\frac{G|\Gamma \Rightarrow \alpha \quad G| \alpha, \Delta \Rightarrow \Pi}{G \mid \Gamma \Rightarrow \Pi}
$$

the induction hypothesis together with Lemma 8.2 yields $\left(\epsilon ; G^{\bullet}\right) \subseteq \Gamma^{\bullet} \multimap \alpha^{\bullet}$ and $\left(\epsilon ; G^{\bullet}\right) \subseteq \alpha^{\bullet} \circ \Delta^{\bullet} \multimap \Pi^{\bullet}$. Hence $\left(\epsilon ; G^{\bullet}\right) \circ \Gamma^{\bullet} \subseteq \alpha^{\bullet}$ and $\left(\epsilon ; G^{\bullet}\right) \circ \alpha^{\bullet} \subseteq \Delta^{\bullet} \multimap \Pi^{\bullet}$. From this, we derive $\left(\epsilon ; G^{\bullet}\right) \circ\left(\epsilon ; G^{\bullet}\right) \subseteq \Gamma^{\bullet} \circ \Delta^{\bullet} \multimap \Pi^{\bullet}$. Since $\left(\epsilon ; G^{\bullet}\right) \circ\left(\epsilon ; G^{\bullet}\right)=\left(\epsilon ; G^{\bullet} \mid G^{\bullet}\right)=\left(\epsilon ;(G \mid G)^{\bullet}\right)$, Lemma 8.2 yields $\vdash_{\text {HFLe }+R}^{c f}(G|G| \Gamma, \Delta \Rightarrow \Pi)^{\bullet}$. By $(E C)$, we obtain $\vdash_{\text {HFLe }+R}^{c f}(G \mid \Gamma, \Delta \Rightarrow \Pi)^{\bullet}$.

Suppose now that the derivation ends with an instance of a completed hyperstructural rule $(r) \in R$. For simplicity, 
we assume that it is of the form:

$$
\frac{G\left|S_{1} \cdots G\right| S_{n}}{G\left|\Gamma_{1}, \ldots, \Gamma_{n}, \Lambda \Rightarrow \Pi\right| \Gamma_{n+1}, \ldots, \Gamma_{m} \Rightarrow}
$$

By the coupling and strong subformula properties, each $S_{i}$ must be either of the form (1) $\Gamma_{i_{1}}, \ldots, \Gamma_{i_{k}}, \Lambda \Rightarrow \Pi$, or of the form (2) $\Gamma_{i_{1}}, \ldots, \Gamma_{i_{k}} \Rightarrow$ with $1 \leq i_{1}, \ldots, i_{k} \leq m$.

Now, let $F_{0} \in G^{\bullet},\left(\Delta_{1} ; H_{1}\right) \in \Gamma_{1}^{\bullet}, \ldots,\left(\Delta_{m} ; H_{m}\right) \in$ $\Gamma_{m}^{\bullet},\left(\Sigma_{1} ; F_{1}\right) \in \Lambda^{\bullet}$ and $\left(\Sigma_{2} \Rightarrow \Theta ; F_{2}\right) \in \Pi^{\bullet \triangleright}$. Our purpose is to show that

$$
H\left|\Delta_{1}, \ldots, \Delta_{n}, \Sigma \Rightarrow \Theta\right| \Delta_{n+1}, \ldots, \Delta_{m} \Rightarrow
$$

is cut-free provable in HFLe $+R$, where $H=$ $\left(F_{0}\left|F_{1}\right| F_{2}\left|H_{1}\right| \cdots \mid H_{m}\right)$ and $\Sigma=\Sigma_{1}, \Sigma_{2}$.

For each $1 \leq i \leq n$, let $S_{i}^{*}=\Delta_{i_{1}}, \ldots, \Delta_{i_{k}}, \Sigma \Rightarrow \Theta$ and $H_{i}^{*}=\left(F_{0}\left|F_{1}\right| F_{2}\left|H_{i_{1}}\right| \cdots \mid H_{i_{k}}\right)$ if $S_{i}$ is of type (1) above, and $S_{i}^{*}=\Delta_{i_{1}}, \ldots, \Delta_{i_{k}} \Rightarrow$ and $H_{i}^{*}=$ $\left(F_{0}\left|H_{i_{1}}\right| \cdots \mid H_{i_{k}}\right)$ if $S_{i}$ is of type (2). It is not hard to see that

$$
\frac{H\left|S_{1}^{*} \cdots, H\right| S_{n}^{*}}{H\left|\Delta_{1}, \ldots, \Delta_{n}, \Sigma \Rightarrow \Theta\right| \Delta_{n+1}, \ldots, \Delta_{m} \Rightarrow}
$$

is a correct instance of $(r)$; notice in particular that there is no matching constraint for the conclusion because of the linear-conclusion property. ${ }^{4}$ Since $H_{i}^{*} \mid S_{i}^{*} \in\left(G \mid S_{i}\right)^{\bullet}$ for every $1 \leq i \leq n$, the induction hypothesis and $(E W)$ imply that the conclusion is cut-free provable.

Let us now consider a valuation given by $p^{\bullet}=(p ; \emptyset)^{\triangleright \triangleleft}$. Under this specific valuation, we have the following form of Okada's lemma [13]:

Lemma 8.4. For any formula $\alpha,(\alpha ; \emptyset) \in \alpha^{\bullet} \subseteq(\Rightarrow \alpha ; \emptyset)^{\triangleleft}$.

Proof. By induction on the structure of $\alpha$. The case $\alpha=p$ follows by the identity axiom.

Suppose that $\alpha=\beta \rightarrow \gamma$. To show that $(\beta \rightarrow \gamma ; \emptyset) \in$ $\beta^{\bullet} \multimap \gamma^{\bullet}$, let $(\Gamma ; G) \in \beta^{\bullet}$ and $(\Delta \Rightarrow \Pi ; H) \in \gamma^{\bullet \triangleright}$. The induction hypotheses $\beta^{\bullet} \subseteq(\Rightarrow \beta ; \emptyset)^{\triangleleft}$ and $(\gamma ; \emptyset) \in \gamma^{\bullet}$ imply that $\Gamma \Rightarrow \beta \mid G$ and $\gamma, \Delta \Rightarrow \Pi \mid H$ are cut-free provable. Hence so is $\Gamma, \beta \rightarrow \gamma, \Delta \Rightarrow \Pi|G| H$. This proves $(\beta \rightarrow \gamma ; \emptyset) \in \beta^{\bullet} \multimap\left(\gamma^{\bullet \triangleright \triangleleft}\right)=\beta^{\bullet} \multimap \gamma^{\bullet}$.

To show that $\beta^{\bullet} \multimap \gamma^{\bullet} \subseteq(\Rightarrow \beta \rightarrow \gamma ; \emptyset)^{\triangleleft}$, let $(\Gamma ; G) \in$ $\beta^{\bullet} \multimap \gamma^{\bullet}$. The induction hypothesis $(\beta ; \emptyset) \in \beta^{\bullet}$ implies $(\beta, \Gamma ; G) \in \gamma^{\bullet} \subseteq(\Rightarrow \gamma ; \emptyset)^{\triangleleft}$. Hence $\beta, \Gamma \Rightarrow \gamma \mid G$ is cutfree provable and so is $\Gamma \Rightarrow \beta \rightarrow \gamma \mid G$. This proves the claim. The other cases are similar.

Theorem 8.5 (Completeness). For any hypersequent $G$, we have $G \in G^{\bullet}$ under the valuation $p^{\bullet}=(p ; \emptyset)^{\triangleright \triangleleft}$. Hence if $(\epsilon ; \emptyset) \in \Gamma^{\bullet} \multimap \Pi^{\bullet}$, then $\Gamma \Rightarrow \Pi$ is cut-free provable in HFLe $+R$

\footnotetext{
${ }^{4}$ It is instructive to try to prove soundness for HFLe $+\left(S_{I}\right)$ (see Section 6). The argument would break down precisely at this point, due to lack of the linear-conclusion and coupling properties.
}

Proof. For any sequent $S=(\Gamma \Rightarrow \Pi)$, we have $(\Gamma ; \emptyset) \in$ $\Gamma^{\bullet}$ and $\Pi^{\bullet} \subseteq(\Rightarrow \Pi ; \emptyset)^{\triangleleft}$ by Lemma 8.4 (and by the definition of $\mathbf{0}$ when $\Pi$ is empty). The latter implies ( $\Rightarrow$ $\Pi ; \emptyset) \in \Pi^{\bullet \triangleright}$, hence $\Gamma \Rightarrow \Pi \in S^{\bullet}$, and so $G \in G^{\bullet}$ for any hypersequent $G$.

Corollary 8.6 (Uniform cut-elimination). Let $R$ be a set of completed hyperstructural rules. If $\vdash_{\mathbf{H F L e}+R} G$, then $G$ is cut-free provable in HFLe $+R$.

Proof. Follows by Theorems 8.3 and 8.5.

Remark 8.7. The lattice reduct of the algebra $\mathbf{A}$ is complete. Hence Corollary 8.6 can be easily extended to predicate logics. Extensions to higher order logics and noncommutative ones are also easy.

Corollary 8.8 (Uniform algebraic completeness). Suppose that $R$ is equivalent in HFLe to a set $K$ of axioms. A formula $\alpha$ is valid in every $F L_{e}$-algebra satisfying $K$ if and only if $\alpha$ is provable in HFLe $+R$.

Proof. $(\Rightarrow)$ Since $\vdash_{\text {HFLe }+R} K$, Theorem 8.3 implies that A satisfies $K$. Hence by assumption $(\epsilon ; \emptyset) \in \alpha^{\bullet}$. The claim follows by completeness. $(\Leftarrow) \vdash_{\text {HFLe }+R} \alpha$ implies $\vdash_{\text {FLe }+K} \alpha$. The claim then follows by the soundness of FLe.

To illustrate the use of our results, let WNM be the fuzzy logic defined in [6] as FLew + (prelinearity) + (weak nilpotent minimum) (see Example 3.2). Theorems 5.6, 7.1(b) and Corollary 8.6 automatically yield:

Corollary 8.9. The hypersequent calculus obtained by extending HFLew with (com) and the rule (wnm) of Example 6.5 is a cut-free calculus for WNM.

\section{Conclusion}

We introduce an algorithm that generates equivalent structural rules, in sequent and hypersequent calculi, from a large class of (Hilbert) axioms. The key idea for determining when this is possible is the identification of a hierarchy of formulas $\mathcal{P}_{n}, \mathcal{N}_{n}$ - similar to the arithmetic hierarchy $\Sigma_{n}, \Pi_{n}-$ which keeps track of polarity alternation (cf. [1]). We show how to transform

1. any axiom in $\mathcal{N}_{2}$ into an equivalent set of (sequent) structural rules, and

2. any axiom in $\mathcal{P}_{3}^{\prime}\left(\subseteq \mathcal{P}_{3}\right)$ into an equivalent set of hyperstructural rules.

If the generated rules are acyclic, they are further transformed (completed) into equivalent analytic rules. This also holds when the base calculus contains weakening, in which 
case the automated transformation of axioms into equivalent sets of analytic hyperstructural rules applies to the whole class $\mathcal{P}_{3}$.

Every hypersequent calculus defined by extending HFLe with a set of completed rules is shown to enjoy cutadmissibility, via a uniform and semantic proof (the first such for any hypersequent calculus).

Although some particular formulas beyond $\mathcal{N}_{2}$ (resp. $\mathcal{P}_{3}$ ) can be captured by multiple-conclusion (hyper)sequent calculi, as in the case of weak excluded middle in Gentzen LK calculus for classical logic or of Łukasiewicz axiom (see Example 7.4) in the hypersequent calculus for Łukasiewicz logic in [10], we conjecture that the expressive power of single-conclusion sequent (resp. hypersequent) structural rules is limited to $\mathcal{N}_{2}$ (resp. $\mathcal{P}_{3}$ ) formulas.

We conclude the paper by stating the challenging question of identifying the level of generality, beyond hypersequents, appropriate for dealing, in a uniform way, with axioms at levels higher than $\mathcal{P}_{3}$.

\section{References}

[1] J.-M. Andreoli. Logic programming with focusing proofs in linear logic. Journal of Logic and Computation, 2(3):297347, 1992.

[2] A. Avron. Hypersequents, logical consequence and intermediate logics for concurrency. Annals of Mathematics and Artificial Intelligence, 4: 225-248, 1991.

[3] A. Avron. The method of hypersequents in the proof theory of propositional non-classical logics. In W. Hodges et al. editors, Logic: from foundations to applications. Proc. Logic Colloquium, Keele, UK, 1993, pages 1-32, 1996.

[4] M. Baaz, A. Ciabattoni, and F. Montagna. Analytic calculi for monoidal t-norm based logic. Fundamenta Informaticae, 59(4):315-332, 2004.

[5] A. Ciabattoni and M. Ferrari. Hypersequent Calculi for some Intermediate Logics with Bounded Kripke Models. Journal of Logic and Computation, 11(2): 283-294. 2001.

[6] F. Esteva and L. Godo. Monoidal t-norm based Logic: towards a logic for left-continuous t-norms. Fuzzy Sets and Systems, 124: 271-288, 2001.

[7] G. Gentzen. Untersuchungen über das logische schliessen. Math. Zeitschrift, 39:176 -210, 405-431, 1935.

[8] N. Galatos, P. Jipsen, T. Kowalski and H. Ono. Residuated Lattices: an algebraic glimpse at substructural logics. Studies in Logics and the Foundations of Mathematics, Elsevier, 2007.

[9] R. Hori, H. Ono, and H. Schellinx. Extending intuitionistic linear logic with knotted structural rules. Notre Dame Journal of Formal Logic, 35(2): 219-242, 1994.

[10] G. Metcalfe, N. Olivetti and D. Gabbay. Sequent and Hypersequent Calculi for Abelian and Łukasiewicz Logics. ACM TOCL. 6(3): 578-613, 2005.

[11] G. Metcalfe and F. Montagna. Substructural Fuzzy Logics. Journal of Symbolic Logic 72(3): 834-864, 2007.
[12] M. Ohnishi and K. Matsumoto. A system for strict implication. Annals of the Japan Association for Philosophy of Science, 2:183-188, 1964.

[13] M. Okada. A uniform semantic proof for cut-elimination and completeness of various first and higher order logics. Theoretical Computer Science, 281:471-498, 2002.

[14] H. Ono. Semantics for Substructural Logics. In P. SchroederHeister and K. Došen ed. Substructural Logics, 259-291. 1993.

[15] F. Belardinelli, H. Ono and P. Jipsen. Algebraic aspects of cut elimination. Studia Logica, 68:1-32, 2001.

[16] A. Prijately. Bounded Contraction and Gentzen style Formulation of Łukasiewicz Logics. Studia Logica, 57: 437-456, 1996.

[17] G. Sambin, G. Battilotti and C. Faggian, Basic logic: reflection, symmetry, visibility. Journal of Symbolic Logic, 65: 979-1013, 2000.

[18] K. Terui. Which Structural Rules Admit Cut Elimination? An Algebraic Criterion. Journal of Symbolic Logic, 72(3): 738-754, 2007. 